\title{
REFLEXIONANDO Y CUESTIONANDO LA POLÍTICA SANITARIA EN AMÉRICA LATINA: IMPLICACIONES EN INVESTIGACIÓN EN LA ENFERMERÍA COMUNITARIA, 1980 - 2000
}

\author{
Reflecting and questioning the Sanitary Politics in Latin America: \\ Implications in Research in the Communitarian Nursing, $1980-2000$ \\ Refletindo e questionando a Política Sanitária na América Latina: \\ Implicações na Pesquisa em Enfermagem Comunitária, 1980 - 2000
}

Kelly Myriam Jiménez de Aliaga
María Guadalupe Vásquez Salazar

\author{
Cinthya Patricia Ibarra González \\ María Guadalupe Vásquez Treviño
}

Olga Lidia Banda González

Maria Ramona Medrano Martínez

\section{Resumen}

El objeto de estudio: la vinculación analítica intersectorial y procesos que inciden en la política sanitaria en América Latina, significado social y el cuidado de enfermería comunitaria. Objetivos: describir la política sanitaria; analizar la práctica de Enfermería en diferentes niveles de complejidad, atención, participación y discutir su práctica comunitaria para implementar políticas sanitarias. Referencial teórico, ideología: Thompson 1995, cultura: Geertz 1989. Reflexión analítica, dialéctica, en implementación de programas y reformas, a través de revisión documentaria y estudios. Investigación cualitativa. Sujetos: enfermeras. Se evidenciaron: reformulaciones normativas verticales permanentes, realidad social con problemas convencionales, emergentes que alteran el bienestar comunitario. Concluimos que la enfermería precisa, investigar críticamente: normas sanitarias, el cuidado que ofrece, su interdisciplinaridad e interacción social, resguardando derechos humanos ante normas discriminadoras, situación conflictiva por incoherencias entre directrices institucionales verticales y autonomía profesional, con perspectiva de crear Líneas de Investigación en Enfermería de cuestionamiento crítico de Política Sanitaria en América Latina.

Palabras clave: Enfermería en Salud Comunitaria. Investigación en Enfermería. Política de Salud.

\begin{abstract}
The study object: the intersectorial analytical entailment and processes that affect the sanitary policy in Latin America, social meaning and the care of communitarian nursing. Objectives: to describe the sanitary policy; to analyze the practice of nursing in different levels from complexity, attention, participation and to discuss its communitarian practice to implement political toilets. Referential theoretician, ideology: Thompson 1995, culture: Geertz 1989. Analytical, dialectic reflection, in implementation of programs and reforms, through documentaria revision and studies. Qualitative investigation. Subjects: nurses. They demonstrated themselves: permanent vertical normative reformulaciones, social reality with conventional, emergent problems that alter the communitarian well-being. We concluded that precise nursing, to investigate critically: sanitary norms, the care that offers, its interdisciplinaridad and social interaction, protecting human rights before discriminating norms, conflicting situation by incoherences between vertical institutional directives and professional autonomy, with perspective to create Lines of Investigation in Nursing of critical cuestionamiento of Sanitary Policy in Latin America.
\end{abstract}

Keywords: Community Health Nursing. Nursing Research. Public Health.

\section{Resumo}

0 objeto do estudo: a vinculação analítica e os processos intersectoriais e processos que incidem na política sanitária em América Latina, significado social e o cuidado de enfermagem comunitária. Objetivos: descrever a política sanitária; analisar a prática de enfermagem nos níveis diferentes de complexidade, atendimento, participação e discutir sua prática comunitária para a implementação da política sanitária. 0 referencial teórico, ideologia: Thompson 1995, cultura: Geertz 1989. Reflexão analítica, dialética, na implementação de programas e das reformas, através de revisão documentaria e estudos. Investigação Qualitativa. Sujeitos: enfermeiras. Evidenciaram-se: reformulações normativas verticais permanentes, realidade social com problemas convencionais, emergentes que alteram o bem estar comunitário. Nós concluímos que a enfermagem precisa, pesquisar criticamente: normas sanitárias, o cuidado que oferece, sua interdisciplinaridade e interação social, protegendo direitos humanos diante das normas discriminadoras, situação conflitiva pelas incoerências entre diretrizes institucionais verticais e a autonomia profissional, na perspectiva de criar linhas de pesquisa na enfermagem de questionamento crítico da Política Sanitária em América Latina.

Palabras clave: Enfermagem em Saúde Comunitária. Pesquisa em Enfermagem. Política da Saúde. 


\section{INTRODUCCIÓN}

La Investigación en Enfermería Comunitaria, aborda a través de una visión crítica y reflexiva las Políticas de Salud en América Latina y las implicaciones para el ejercicio profesional de forma integrada e interdisciplinaria; estudia las intervenciones de enfermería en la salud de los individuos, familias y comunidad, enfocando más allá de los aspectos biopsicosociales los aspectos demográficos, epidemiológicos, la diversidad de costumbres, creencias y culturas en salud, entre otros.

Orienta su visión en la salud de los individuos, como actores sociales en interrelación con grupos específicos, como sujetos de investigación, que primeramente, son constituidos teóricamente como componentes del objeto de estudio; en el campo hacen parte de una relación de la ínter subjetividad y de la interacción social con el investigador, dando un producto nuevo y confrontante tanto con la realidad concreta como con las hipótesis y supuestos teóricos, en un proceso más amplio de construcción de conocimientos ${ }^{1}$

Teniendo en consideración que el conocimiento se organiza en relación a dos polos: el abstracto puro, por el cual extraemos las estructuras abstractas del conocimiento y el concreto puro por el cual es asegurado en el fundamento de ese conocimiento en la realidad concreta ${ }^{2}$, la investigación científica en Enfermería Comunitaria, por su encuadramiento es socio - cultural se presenta sobrecargada de significado ideológico, en una concepción de evaluación en que todas las formas de pensamiento están socialmente condicionadas por sus misma naturaleza, muchas veces psicológicamente deformada por la presión de emociones personales que requiere de la teoría del conocimiento para tratar del elemento social en el búsqueda y percepción de la verdad ${ }^{3}$, de fenómenos de la realidad comunitaria para transformarla.

\section{ANTECEDENTES}

En los países en desarrollo en el contexto del mundo inmersos en regímenes neoliberales y de globalización, la implementación de nuevos Programas de Política de Salud, traen en su mayoría, graves perjuicios a la mayoría de la población a través de Reformas, propias de otras realidades que fueron traídas para América Latina, en un contexto de liquidación de deudas pendientes y directrices a históricas, deterministas estructurales, que limitan la capacidad de acción de los individuos que no aseguran las condiciones objetivas universales (económicas, políticas y culturales), en las sociedades en que se implantan, para el ejercicio de la libertad de cada ser humano, y por ende no garantiza la vida y la salud.

En el caso peruano la política del sector salud en la década de los noventa se tornó contradictoria; por un lado se han logrado avances en la recuperación y ampliación de la oferta de atención primaria de salud, al mismo tiempo, son pocos los logros en cuanto a la transformación de los sistemas de salud para lograr los objetivos de equidad, eficiencia y calidad que planteó la reforma de salud que aún es una tarea plena pendiente. Las orientaciones iniciales de la reforma estuvieron marcadas por conceptos importados y adaptados a la realidad peruana, presentaron diferencias y conflictos políticos mayores que dominaron el escenario político nacional con opiniones relativas a la política salud; tornándose ésta, uno de los espacios con mejores posibilidades de resolver esta situación, debido a que al igual que en el resto del mundo no existen diferencias ideológicas tan marcadas entre las distintas fuerzas políticas y sociales ${ }^{4}$.

Essa reforma, todavía, según los autores ${ }^{5}$ surge en el contexto de las reformas estructurales iniciadas en los años 1990, en el marco de los cambios ministeriales ocurridos en los años 1991- 1992 en un contexto de ajuste estructural neoliberal, como propuestas estrechamente vinculadas a las orientaciones del Banco Mundial (BM) y del Banco Interamericano de Desarrollo (BID) que influiríon de manera determinante en el gasto social en salud, aunque estas propuestas estén vinculadas a ofrecer nuevo sistema de cuidado colectivo e individual, donde se prioriza el grupo materno infantil, niñez y adolescencia.

En el caso de Brasil, en estudios sobre la inserción de la Enfermería y la evaluación del impacto políticosocial de los Programas Nacionales de Salud MaternoInfantil se resaltan dos aspectos fundamentales identificados en la elaboración de esos programas en los años de 1974, 1977 e 1984: el primero caracteriza el carácter vertical del programa, esto es, el hecho de tener sus metas y normas decididas en nivel central y con criterios técnicos en concordancia con el modelo tecnócrata de la administración brasilera de aquella época, en un modelo de atención que no siempre correspondió a la condición de salud de las poblaciones asistidas; el segundo es relacionado con los instrumentos de operacionalización del programa cuyo manejo requería informaciones que los ejecutores de las actividades programadas no disponían ${ }^{6}$.

En la política de salud en el Brasil en los años 90, a pesar de las dificultades, hubieron avances en el modelo de descentralización adoptado por la Política de Salud 
en dirección a la municipalización de la gestión (incluyendo recursos e instrumentos), así como de las funciones estatales de coordinación del sistema de referencia intermunicipal, ampliando de esta manera el poder inductor y regulador del nivel federal al introducir nuevos mecanismos de transferencia vinculados a las acciones y programas asistenciales ${ }^{7}$.

En el caso de México, existen estudios que definen que las medidas de reforma del sistema mexicano de salud de los años 1995 tenían como objetivos mejorar la calidad, eficiencia y equidad de los servicios públicos, a través de: la introducción de un nuevo esquema financiero dentro del Instituto Mexicano de Seguridad Social, la creación de áreas médicas con asignaciones de presupuesto por edad y sexo, la afiliación de miembros de la economía informal a través de Seguro para la familia, la conclusión de la descentralización de los servicios de salud para los no asegurados y la entrega de un paquete de intervenciones a través del PAC (Programa de Ampliación de Cobertura) a ciudadanos que no tenían acceso a los servicios de salud hasta $1995^{8}$.

Estas medidas tuvieron un impacto positivo en el acceso de los más carentes a la atención de salud, y el autor ${ }^{8}$, contrariamente poco pudieron hacer por la justicia del financiamiento, la mala distribución de recursos, las barreras de género, la segmentación de la atención, la concentración en la atención curativa, la rendición de cuentas, la transparencia; el creciente problema de la violencia y la seguridad pública de las inequidades sanitarias, la segmentación y la creciente concentración de los recursos en la atención curativa del segundo y tercer nivel. Por otro lado se considera que los servicios de salud siguen siendo culturalmente homogéneos contrariamente son impuestos desde arriba se imparte educación en salud partiendo del supuesto que los indios carecen de conocimientos sanitarios ${ }^{9}$.

Se encontraron estudios sobre relaciones políticas y descentralización de la salud Pública del Brasil y México, sobre los procesos de cambios ocurridos en distintas esferas de la política: gobierno federal, instituciones públicas de salud y gobiernos subnacionales y como se vincularon en cada país. En México, las tres etapas se refieren a los tres últimos sexenios del Gobierno Federal, correspondientes a los años del presidente De La Madrid (1982-1988), Salinas (1988-1994) y Zedillo (1994-2000). En Brasil, a su vez, las tres etapas comprenden el último gobierno autoritario - de Figueiredo, los tres primeros gobiernos democráticos, de Sarney, Collor e Itamar, y el Gobierno de Cardoso después de 1994, definiendo que en ambos contextos las transiciones y coaliciones políticas son de naturaleza distinta, hubo influencia del Estado y renacieron las relaciones políticas y económicas del federalismo en cada país ${ }^{10}$.

Según este análisis en México, los Estados pasan a ser piezas claves de la transición en la etapa de Salinas, desde el 82, la transición política es marcada por una liberalización política lenta, con una coalición política comprometida también con la liberalización económica. La descentralización tuvo inicio incipiente, seguido de una interrupción y, al fin, la implementación de la descentralización junto a todos los Estados; mas el centralismo permanece en la estructura fiscal y tributaria, hay poca flexibilidad de presupuestos para que los Estados y municipios se hagan cargo de su papel de intervención local.

En Brasil los Estados son piezas claves de la transición desde su principio, antes del 94 permanece en el poder una coalición política comprometida con democracia y desarrollo, posteriormente a este año hubo compromiso con la democracia y estabilización económica, se dieron paquetes básicos del año 1996. El Sistema Unico de Salud - SUS cambió drásticamente el sistema público de salud, con el surgimiento de un sistema completamente innovador, con nuevos actores, la descentralización se caracterizó por tener autonomía administrativa, política y económico-financiera (diferenciada entre los municipios), controlada por mecanismos financieros relacionados a los programas específicos.

Paralelamente en el Perú, en los años 1982 al 1988, con los gobiernos de Bermúdez y Alan García, el país vivenció la transición de gobierno militar a democrático, aplicación de estrategias económicas de protección estadista a modelos económicos neoliberales de mercado. Las instituciones se caracterizaron por: ampliación de los horarios, discurso de equidad y calidad de atención; improvisación normativa, discrepancias políticas, sin diferencias ideológicas, se reforzó la práctica antidemocrática, con primacía del centralismo.

En los años 90, en el primer gobierno de Fujimoria se asumieron reformas como condición de supervivencia en la forma de políticas de emergencia; la atención de salud fue a través de seguros parciales para grupos específicos, con reformas también parciales sin tener un proyecto global. Como respuesta a esto en las aulas universitarias se recomponen y renuevan los paradigmas y discursos sanitarios con los primeros cursos de maestría en las profesiones de la salud en las Escuelas de pos-grado de la Universidad Cayetano Heredia y de la Universidad Nacional de Trujillo (Maestría en Enfermería con mención en Salud Familiar y Comunitaria), tornándose espacios de reflexión crítica cultural y política.

En los años del 94 al 2000, en el segundo gobierno de Fujimori, se dieron intensos cambios, que 
respondían a prioridades coyunturales carentes de visión estratégica, de consulta técnica y participación de la sociedad civil, crisis de los partidos políticos. Esta situación no garantizó la contribución de actores sociales, por la (in) coherencia del conocimiento, de la práctica y el contexto socio político en salud; entre 1995 y 1998, se formularon los lineamientos de políticas y el modelo de la reforma sectorial del Ministerio de Salud (MINSA); se realizaron las aplicaciones iniciales, en una forma de complementar el segundo momento ${ }^{4}$.

Actualmente se puede concluir que los estudios revisados nos alertan que, en pocas áreas de la economía las relaciones entre el Estado y el mercado de trabajo son tan complejas como en el área de salud, donde los errores del mercado y de las políticas exteriores dan apertura mayor al envolvimiento del sector público, mucho más que en otras áreas. Esto es reforzado por un lado, por la cultura curativa de las políticas de salud en América Latina, donde el ciudadano tiene la posibilidad de comparar costos, calidad, oportunidad, trato de servicios y productos que derivan de una atención de salud por los elevados niveles de morbilidad de dolencias agudas como consecuencia de deficiente saneamiento básico, y sumado a esto las carencias educacionales que no le permite optar por la prioridad de una actitud preventiva. Y por otro lado, por la fácil difusión que los medios de comunicación vehiculan estrategias ideológicas gubernamentales de legitimación de estos programas. a través de la creación de objetos simbólicos lo que es atribuido a objetos y formas simbólicas, que pueden ser estimados por los individuos que los producen y reciben, esto es, por ellos aprobados o condenados, apreciados o despreciados ${ }^{11}$.

Esto es corroborado por estudios sobre herramientas pedagógicas para la gerencia de políticas y programas sociales, donde se estructuran propuestas en torno a una serie de estrategias, como son: la formación e implementación de políticas y programas sociales, que consideran procesos técnicos, científicos y analíticos para el diseño, formulación, aplicación y evaluación de políticas y programas sociales; diseño y aplicación de políticas sociales y programas locales - regionales a favor de distintos grupos de población; a ser incluidos en la agenda pública ${ }^{12}$.

Estos perfiles políticos, tienen repercusión en la implementación de los programas de salud que ante las inconsistencias normativas, la práctica profesional de enfermería comunitaria se torna conflictiva, por las carencias sociales y humanitarias de la comunidad, asumiendo como desafío de la necesidad de envolvernos en el conocimiento y desarrollo de habilidades de investigación comunitaria, con énfasis en salud colectiva, salud pública, a fin de lograr los siguientes, objetivos:

- Describir las Políticas Sociales y de Salud en América Latina reflexionando sobre sus interrelaciones con el proceso de salud enfermedad.

Analizar la práctica de Enfermería en diferentes niveles de complejidad (primario, secundario), de atención (promoción / prevención / curación / rehabilitación) y de intervención (individual / familiar / comunitaria).

Discutir la práctica de Enfermería en la implementación de las políticas sociales y de salud en América Latina.

\section{ENFERMERÍA: \\ CRÍTICA Y AUTOCRÍTICA DE SU PRÁCTICA COMUNITARIA}

La práctica comunitaria de enfermería en América Latina está relacionada a la visión del ejercicio profesional en su relación con la estructura macro social, como el Estado y las políticas sociales, en particular las políticas de salud y de educación, y en el nivel micro en las relaciones que establece con individuos sanos o enfermos, con la comunidad de usuarios o con el equipo de trabajo en la producción de acciones de enfermería.

El profesional de enfermería ha experimentado mayor movilidad en sus funciones y en su preparación que el resto de los trabajadores del área de la salud ${ }^{13}$. Por tal razón, abordar la práctica de enfermería comunitaria, teniendo como objeto de estudio el cuidado de la comunidad, exige vincular el análisis con los principales sectores y procesos que inciden directamente en la determinación de sus características, su significado social y sus funciones específicas. Es ampliamente reconocida la intervención del Estado en la sociedad y particularmente en los sectores como la salud y la educación, pues hace parte de la responsabilidad que le compete; la que para su análisis las enfermeras debemos enfatizar en los aspectos económico, político e ideológico.

Por muchos años, la enfermería no fue tomada en cuenta cuando se adoptaron decisiones importantes en la aplicación de las políticas de salud de los países latinoamericanos, entre ellos el Perú, por ejemplo, ni se le consideraba al diseñar el proceso de planificación de la salud, realidad que se abordó con inteligencia intelectual por parte de los diferentes movimientos profesionales respaldados por la OPS/OMS, que 
instaron a los gobiernos la participación de enfermería en este proceso.

En los años 70 en Latino América con sede en Lima se realizó, la reunión de un Grupo de Trabajo sobre Programación en Enfermería, a la que asistieron 28 enfermeras en representación de ocho países, logrando esbozar las líneas directrices para hacer efectiva la participación de la enfermería en la planificación de salud, respondiendo a la Reunión Extraordinaria del Consejo Económico y Social al Nivel Ministerial, celebrada en Punta del Este, Uruguay, en 1961, que había recomendado a los gobiernos, el Plan Decenal de Salud Pública de la "Alianza para el Progreso" que, entre otras medidas a largo plazo, exhortaba a las autoridades nacionales para dar particular importancia a la formación y capacitación de profesionales y auxiliares para las acciones de prevención y curación de las enfermedades y la urgencia de intensificar la capacitación del personal de enfermería, surgiendo cursos de especialización en enfermería materno infantil y médico quirúrgico, en salud mental y psiquiatría ${ }^{14}$.

Otra recomendación a la OPS/OMS fue establecer un centro para el desarrollo de la tecnología de la enseñanza y la investigación en enfermería ${ }^{15}$, con miras a fortalecer la educación de enfermería como un medio de mejorar la prestación de servicios de salud, la que respondió con la organización y celebración en Costa Rica de un Seminario sobre nuevas Dimensiones en el Papel de la Enfermera en la Prestación de la Atención Primaria que contó con la participación de 19 enfermeras y 17 médicos de 13 países latinoamericanos, donde las enfermeras definieron un nuevo concepto del cuidado de la salud integral que reconoce que la salud del individuo y de la población no solo depende de las acciones del sector salud, sino de las que realizan todos los sectores de la sociedad, reconociéndose que el individuo y la comunidad tienen el derecho de intervenir en el proceso de su propio desarrollo, y que su participación sea activa y decisiva en la obtención y mantenimiento de la salud. En este espacio se analizó la situación de sub utilización profesional de Enfermería, en comparación con su potencial para asumir funciones más directas en la prestación de la atención primaria que antes era privativa del médico ${ }^{14}$

Es así que se determinó que los servicios e intervenciones en la prestación de la enfermera de atención primaria con abordaje de salud integral recaen directamente sobre los individuos, la familia y la comunidad; práctica que ha permitido hasta este momento que la enfermera alcance un grado de competencia que le permite tomar decisiones y compartir responsabilidades con los demás miembros de equipo en la tarea de elevar el nivel de salud de la población a su cargo ${ }^{14}$. Por lo tanto, concientes de las inconsistencias de los programas en cuanto a coberturas de atención, debilidad para hacerla operativa ante los escasos recursos, entre otros; al ejercer la atención primaria sus funciones no solamente se circunscriben al cumplimiento de las normas de los programas oficiales de salud sino también a implementar estos programas, cuando en la práctica no alcanzan a cubrir las expectativas de atención a la salud y del cumplimiento de derechos humanos, por los conflictos permanentes que encuentran entre su práctica profesional y las normas de salud excluyentes ${ }^{16}$.

La enfermería comunitaria en el contexto de sus cuestionamientos del saber, se la puede considerar como el eje de las acciones de promoción de la salud y prevención de enfermedades, velando en el día a día por la calidad de los servicios prestados, y por la relación entre el equipo de salud y la comunidad ${ }^{16}$, lo que nos hace reflexionar en el sentido que el desempeño de la enfermera comunitaria, demanda una supervisión técnica que contribuya a ofrecer cuidado en el área de la salud en el cumplimiento crítico, de las metas de los programas de salud orientadas a ofrecer una prestación libre de riesgos, y que sus acciones ejecutadas tengan carácter transformador, en un ejercicio dinámico de sus propios cuestionamientos, éticos, técnicos, científicos, epistemológicos, culturales entre otros ${ }^{16}$.

Posteriormente en los años 80 la OPS/OMS reafirmó la responsabilidad de la enfermería como coordinadora de las acciones de salud en el primer nivel de atención del sistema de salud, definido por un grupo de estudios de profesionales de la salud. Concomitantemente, en la misma época se iniciaron reformulaciones curriculares orientadas hacia la salud de la comunidad y la preparación de las enfermeras para la atención primaria, enfatizando en la articulación docenteasistencial, la educación informal, la autoinstrucción, la enseñanza extra mural, el servicio social para las recién egresadas, entre otros ${ }^{14}$.

En esta época en la XVIII Conferencia Sanitaria Panamericana de la Salud se aprobaron, por primera vez, sendas resoluciones enfatizando la importancia que se debía conceder a la enfermería, instando a los gobiernos miembros a establecer sistemas de información que definieran las esferas de su responsabilidad, la cantidad y categoría del personal necesario compatible con los objetivos del programa local de salud, y la clase y nivel del cuidado de salud que se había de facilitar. ${ }^{17}$ 
Por su lado, la enfermera comunitaria, profesionalmente asume un posicionamiento según su competencia; históricamente, ella ejerció y ejerce un rol comunitario fuera de los muros asistenciales, y siendo enfermera de servicio, por su identidad profesional asume el rol de enfermera comunitaria en el contexto de su hogar, de su vecindario; matizando su función comunitaria, a la hora de empatizar con los problemas colectivos sanitarios cuando en su diario vivir promociona la salud ante la violación de la práctica de estilos de vida saludable; asumiendo inmediatamente dos nobles responsabilidades: la ciudadana y la profesional en una actitud esencialmente de naturaleza educativa.

Su ciencia es el cuidado, en una función de trabajo con grupos para contribuir a enlazar a la comunidad con el sistema institucional de salud, con el objeto de mejorar el nivel de salud de la población inculcando hábitos y prácticas saludables; su método de trabajo es la visita al hogar para enseñar al grupo familiar cómo cuidar su salud mediante las entrevistas y las demostraciones, a la vez que esto le permite relacionarse con las diversas organizaciones que trabajan en pro del bienestar social.

Define como unidad de cuidado a la familia, que constituye el núcleo de la sociedad; está preparada conceptual y vocacionalmente para socializar con las creencias y prácticas de salud de los distintos grupos de la comunidad, conoce la realidad sanitaria y el ámbito de vida de los hogares que visita; está capacitada para reconocer alteraciones del bienestar del individuo familia y comunidad para llamar la atención sobre ellas en el momento preciso, para realizar con criterio científico - técnico los cuidados de enfermería y/o tratamientos prescritos; para orientar al grupo social hacia la promoción y prestación de la salud ${ }^{18}$.

La enfermera comunitaria trabaja muy cerca de la comunidad, conoce los problemas biológicos, sociales, culturales y familiares del sector de población asignado a su cuidado, y siempre está tratando de ayudar a solucionar las dificultades existentes, se puede afirmar que ella es la representante más conocida de las instituciones de salud porque trabaja con los usuarios en los servicios de salud, entrevistándolos individualmente para aclarar sus dudas, para educar en salud; constituye el agente más útil del centro de salud para la enseñanza individual y de la comunidad en los principios de la vida sana y para atraer a los grupos sociales a los servicios de salud ${ }^{17}$.

Considerando que la profesión de enfermería se da principalmente, en el plano del saber de la salud, de la sociología y de la pedagogía, en el plano social y de la cultura, nuestro desafío es la investigación en Enfermería Comunitaria; lo que entendemos por pensar y hacer, a partir de experiencias prácticas y teóricas de enseñar el cuidado a la comunidad y de cuidar mismo; se destaca la importancia de pensar el ser comunitario del cuidado como el de la historia, el de la cultura, el del deseo y de la necesidad de exigencia de pensar la enfermería a partir de otros referenciales, más allá de la biología que sólo centra en la enfermería, el interés en la enfermedad, y no en la salud. Cuidar en salud, da lugar a un nuevo paradigma científico para enfermería, fundamentado, en lo que Florence Nightingale nos dejó como legado ${ }^{19}$.

A nuestro entender la cultura del cuidado ocurre en una ecología que incluye: contexto, interacciones personales e subjetividad humana; en un espacio de expresión que las enfermeras tienen para pensar y hacer su arte de cuidar, considerando la concepción antropológica que considera al hombre atado a telas de significados que él mismo teje las que son asumidas por la cultura cuyo análisis es la conciencia interpretativa a la procura de significados; pues, la cultura de las comunidades, esta localizada en la mente y en el corazón de los hombres; la cultura es pública porque el significado es público; la cultura consiste en estructuras de significado socialmente establecidas ...Un padrón de significados históricamente transmitidos, incorporados a los símbolos, y que relaciona con la noción de texto que desempeña un papel central en su abordaje, y ve los fenómenos culturales como constructos significativos, como formas simbólicas. El análisis de la cultura es entendida como la interpretación de los padrones de significado incorporados a esas formas ${ }^{3}$

La práctica teórica comunitaria de enfermería, en el día a día, es escenario de contradicciones entre las normas originadas de las políticas de salud y de la realidad de su práctica, como formas de conflicto en un movimiento dinámico histórico, percibe carencias que le generan conflictos internos, que sumados a las limitaciones de recursos para los usuarios, poco puede conciliar sus pensamientos con argumentación, menos llegar a entender el plano superior de aproximación ética de las normas verticales recibidas de los niveles superiores.

La enfermera en estos conflictos, según los estudios de Aliaga, realizados en el Hospital Belén de Trujillo Perú en 2003, concluyó, que la enfermera asume un posicionamiento crítico y humano en lo que se refiere al cuidado, donde se permean confrontaciones en la 
práctica advenida de los directrices gubernamentales verticales; de carencia de diferentes recursos; de la presión de las necesidades urgentes de los escolares en este caso, que llevan a la enfermera, a veces, a transgredir las normas, en vez de violar sus principios profesionales ${ }^{16}$.

Se puede incrementar, que esse estudio, es ya un nuevo motivo de conflicto, que forma parte del desarrollo histórico e intelectual de la enfermera en la búsqueda de aproximarse cada vez más a la verdad de su práctica teórica.

En la práctica profesional de la enfermera, se puede observar la contradicción de su rol, por un lado, como agente controlador al servicio de las instituciones de salud y de las políticas que determinan su práctica profesional. Por otro lado, la propuesta de la enfermera como agente transformador, actitudes profesionales manifestadas como fenómenos culturales implicados en relaciones de poder y conflicto, lo que la llevó a la autora formular la tesis doctoral que las enfermeras desenvuelven estrategias que instrumentalizan sus actividades profesionales en la implementación de estos programas ${ }^{16}$.

Por su lado la comunidad frente a su realidad tiene una actitud primordial e inmediata de un equipo de individuos que actúan objetiva e prácticamente, de cada individuo histórico que ejerce su actividad práctica en el trato con sus problemas de salud y con los miembros del equipo de salud, teniendo en vista la consecución de los propios fines e intereses, dentro de un determinado conjunto de relaciones sociales, que en la mayoría de veces se torna espacios de insatisfacciones, conflictos e impotencias. Por tanto la realidad se presenta como el campo en que se ejercita su actividad práctica- sensible, sobre cuyo fundamento surgirá la intuición práctica de la realidad ${ }^{20}$.

Esto nos lleva a definir que la profesión de enfermería precisa consolidar su posición dentro del equipo de salud y compartirlos con otras disciplinas, estableciendo compromisos consigo misma y con la sociedad, en defensa de los derechos humanos ante las normas que discriminan al sujeto de nuestra atención aun en escenarios limitantes donde existen grandes diferencias entre la práctica institucional establecida y la necesidad permanente de una práctica profesional con autonomía.

La generación de conflicto permanente en la práctica asistencial de las enfermeras es evidente y a la vez transformadora, pues ellas evidencian hechos, procesos, actitudes que indican y revelan la existencia manifiesta de fenómenos, de modo inadecuado, parcial 0 apenas sobre ciertos ángulos y aspectos, que revelan su movimiento demostrando que no es inerte ni pasivo. En este espacio los fenómenos se dan de una manera dinámica, y al mismo tiempo, se esconden, más vive en los cuestionamientos entre los individuos, como conflictos dinámicos que quedarán registrados en la historia ${ }^{20}$, en lo que se refiere a la actitud de la enfermera ante las carencias, en sus oposiciones 0 resignaciones; opta po el desafío de tomar decisiones, está presente, sea como agente controlador de las normas o transformador en la práctica de sus principios profesionales donde impera la defensa de los derechos humanos.

Esta reflexión debe convocarnos a las enfermeras a asumir un espíritu de investigación profesional, considerando el elevado nivel de conocimiento de la realidad de su práctica que garantiza el conocimiento concreto, teniendo en consideración que el espíritu científico impide que tengamos una opinión sobre cuestiones que no comprendemos, sobre cuestiones que no sabemos formular con claridad, para no incrustar en el nuevo conocimiento un obstáculo epistemológico ${ }^{21}$.

Consideramos que ante éstos hechos la enfermera descubre que debe estudiar la realidad de su práctica profesional para ser entendida desde su óptica, en el conocimiento de las partes de los Programas y Proyecto que le toca implementar, expresados en su totalidad, empezando por analizar la ideología gubernamental de dar prioridad a los grupos de población en riesgo, que ante las inconsistencias en la realidad no se alcanza ofrecer atención ni a estos grupos, por ello es importante citar estudios ${ }^{22}$ que inviten a reflexionar sobre los tipos de pobreza, considerando a los países donde se sufre y se sufrió prolongados periodos de violencia, donde surgió un tipo especifico de pobreza derivada de este fenómeno (población marginada por situaciones de guerra civil, violencia de narcotráfico u otras situaciones similares, caso de Nicaragua, Perú y Guatemala) $)^{22}$.

Ante esto es importante tomar conciencia, que la enfermería convive, participa, comparte vivencias con innumerables formas de insatisfacción comunitaria en su extensión familiar-individual, a las que ofrece la práctica-teórica de la ciencia del cuidado en todas sus dimensiones, por lo que al referir a las pobrezas, no solo a su concepto singular, sino a cualquier necesidad humana fundamental que no es adecuadamente satisfecha. Revela una pobreza humana: la pobreza 
de subsistencia (debido a alimentación y abrigo insuficientes); de protección (debido a sistemas de salud ineficientes, a la violencia, la carrera armamentista, etc.); de afecto (debido al autoritarismo, la opresión, las relaciones de explotación con el medio ambiente natural, etc.); de entendimiento (debido a la deficiente calidad de la educación); de participación (debido a la marginación y discriminación de mujeres, niños y minorías); de identidad (debido a la imposición de valores extraños a culturas locales y regionales, emigración forzada, exilio político, etc.) y así sucesivamente, se podrían denominar pobrezas, pues son mucho más que eso; cada una de ellas genera carencia de bienestar, toda vez que rebasa límites críticos de intensidad y duración ${ }^{23}$.

\section{DISCUSIÓN}

Los lineamientos de Política de Salud en América Latina, en las 2 últimas décadas del siglo XX, tienen implicaciones en cuanto a eficiencia, efectividad y equidad en los sistemas de salud, evidenciándose muchas contradicciones en el aumento de la eficiencia y: disminución de la equidad y acceso a los costos, pérdida de una atención integral y acceso a ella, aumento de desigualdades en cuanto a financiamiento y provisión de recursos.

Las orientaciones iniciales de la reforma presentaron diferencias y conflictos políticos mayores que dominaron el escenario político nacional con opiniones relativas a la política salud; surge en el marco de las reformas estructurales, en un contexto neoliberal, como propuestas vinculadas a las orientaciones del Banco Mundial (BM) y del Banco Interamericano de Desarrollo (BID) que influyen de manera determinante en el gasto social en salud.

Los programas se caracterizaron por ser verticales, modelo tecnócrata de la administración latino americana, modelos de atención que no siempre corresponden a la condición de salud de las poblaciones asistidas; así mismo la no disponibilidad de información previa para la operacionalización del programa.

Se pueden considerar como avances la descentralización adoptada en dirección a la municipalización de la gestión, de las funciones estatales de coordinación del sistema de referencia intermunicipal, ampliando de esta manera el poder inductor y regulador del nivel federal al introducir nuevos mecanismos de transferencia vinculados a las acciones y programas asistenciales, contrariamente poco pudieron hacer por la justicia del financiamiento, la mala distribución de recursos, las barreras de género, la segmentación de la atención, la concentración en la atención curativa, la rendición de cuentas, la transparencia; el creciente problema de la violencia y la seguridad pública las inequidades sanitarias, la segmentación y la creciente concentración de los recursos en la atención curativa del segundo y tercer nivel.

Hubo influencia del Estado y renacieron las relaciones políticas y económicas del federalismo en cada país, cada uno en sus propias características, mas el centralismo permanece en la estructura fiscal y tributaria, con poca flexibilidad de presupuestos para que los Estados y municipios se hagan cargo de su papel de responsabilidad local.

Los sistemas de seguros en salud en las modalidades de SUS, SIS, SP, cambiaron drásticamente el sistema público de salud, con el surgimiento de un sistema completamente innovador, con nuevos actores, la descentralización se caracterizó por tener autonomía administrativa, política y económico-financiera (diferenciada entre los municipios), controlada por mecanismos financieros relacionados a los programas específicos.

\section{CONSIDERACIONES FINALES}

Siendo la equidad y justicia social los elementos primordiales de ciudadanía, para enfermería es un desafío participar en pro de esta consolidación ciudadana, a través de la investigación comunitaria en las diferentes regiones donde nos toca desenvolvernos profesionalmente, por ello el interés sobre la política de salud en los diferentes países de América Latina, cuya reforma se dió como formas de políticas de emergencia; la atención de salud fue a través de seguros parciales, universales o integrales, los que dejan de serlo al determinarse coberturas de atención sea por grupos vulnerables, excepción de atención de ciertas dolencias, exámenes auxiliares, o la necesidad de realizar pagos de inscripción; con reformas también parciales sin tener un proyecto global.

Los intensos cambios en este contexto, responden a prioridades coyunturales carentes de visión estratégica, de consulta técnica y participación de la sociedad civil, que no garantizó la contribución de actores sociales, por la (in) coherencia del conocimiento, de la práctica y el contexto socio político en salud. De esta forma, se puede definir que en pocas áreas de la economía las relaciones entre el Estado y el mercado de trabajo son tan complejas como en el área de salud, donde los 
errores del mercado y de las políticas exteriores dan apertura mayor al envolvimiento del sector público, mucho más que en otras áreas, lo que es reforzado por un lado, por la cultura curativa de las Políticas de Salud en América Latina, donde el ciudadano tiene la posibilidad de comparar costos, calidad, oportunidad, trato servicios y productos que derivan de una atención de salud por los elevados niveles de morbilidad de dolencias agudas y crónicas como consecuencia de deficiente saneamiento básico, y sumado a esto las carencias educacionales que no permite al ciudadano latino americano optar por la prioridad de una actitud preventiva y por otro lado, por la fácil difusión que los medios de comunicación vehiculan estrategias ideológicas gubernamentales de legitimación de estos programas a través de la creación de objetos simbólicos.

Lo expuesto anteriormente referido a los perfiles políticos, tienen repercusión en la implementación de los programas de salud que ante las inconsistencias normativas, la práctica profesional de enfermería comunitaria se torna conflictiva, por las carencias sociales y humanitarias de la comunidad, asumiendo como desafío la necesidad de envolvernos en el conocimiento y desarrollo de habilidades de investigación comunitaria, con énfasis en salud colectiva, salud pública, lo que demanda la oferta de cuidado de enfermería comunitaria en el área de la salud en el cumplimiento crítico, de las metas de los programas de salud orientadas a ofrecer una prestación libre de riesgos, y que sus acciones ejecutadas tengan carácter transformador, en un ejercicio dinámico de sus propios cuestionamientos, éticos, técnicos, científicos, epistemológicos, culturales entre otros.

La actitud de la enfermera ante las carencias, en sus oposiciones o resignaciones está presente, sea como agente controlador de las normas o transformador en la práctica de sus principios profesionales donde impera la defensa de los derechos humanos.

Esta reflexión debe convocarnos a las enfermeras a asumir un espíritu de investigación profesional, considerando el elevado nivel de conocimiento de la realidad de su práctica que garantiza el conocimiento concreto, teniendo en consideración que el espíritu científico impide que tengamos una opinión sobre cuestiones que no comprendemos, sobre cuestiones que no sabemos formular con claridad, para no incrustar en el nuevo conocimiento un obstáculo epistemológico

Finalizando, acreditamos que estas reflexiones teóricas, serán un punto de partida para otros estudios que produzcan conocimientos a cerca de este asunto, en la perspectiva de posibilitar a creación de Líneas de Investigación en Enfermería concordantes con los fenómenos de la realidad de la Política de Salud delos Países de América Latina, que requieren la participación argumentativa inmediata a través de la Investigación en Enfermería Comunitaria.

\section{Referências}

1. Minayo MC. 0 desafio do conhecimento: pesquisa qualitativa em saúde. São Paulo-Rio de Janeiro: Hucitec / Abrasco. 2000.

2. Japiassu H. Introdução ao pensamento epistemológico. $7^{a}$ ed. Rio de Janeiro: 1992; 188-90

3. Geertz C. A interpretação das culturas. 2a. edición. Rio de Janeiro: Editorial Vozes, 1989

4. Arroyo J. Salud: la reforma silenciosa. Perú: Universidad Peruana Cayetano Heredia. 2000; 31-32.

5. Ugarte 0, Monje J. Políticas sociales en el perú: nuevos aportes equidad y reforma en el sector salud. Peru: Instituto de Estúdios Peruanos, 2001; 571-72.

6. Tyrrell MAR. Programas nacionais de saúde materno-infantil impacto político-social e inserção da enfermagem. Esc Anna Nery Rev Enferm 1997; 1:119-134

7. Lescovitz E, Díaz L, Viera C. A política de saúde nos anos 90: relações inter goverrnamentais e o papel das normas operacionais básicas. Rev Ciência \& Saúde; 2001.

8. Duarte G, Brachet M, Campos N, George J; Teorias de enfermagem. Porto Alegre: Artes Médicas; 1993.

9. Cunha F. Equidad y calidad en salud. México: Reforma de los Sistemas de Salud. X Congreso de Investigación en Salud Pública; Trabajo Libre TL007; 2003.

10. Gomes 0, Gómez J, Inclán C. Salud pública de México / la equidad y la imparcialidad en la reforma del sistema mexicano de salud. Rev Salud Pública. 2004; 46(5). 5).

11. Thompson, J. Ideologia e cultura moderna, teoria social crítica na era dos meios de comunicação de massa. 3a. edición. Río de Janeiro(RJ): Vozes; 1995.

12. Francke P. Resumen propuestas para una política de salud. Consorcio de Investigación Económica y social. Perú; 2001

13. Castrillón C, Correa A. La formación universitaria de enfermeras en colombia (1937-1980). Medellín: Universidad de Antioquia, Facultad de Educación; 1985.94-95.

14. Organización Panamericana de la Salud, Organización Mundial de la Salud. El papel de la enfermera en la atención primaria de salud Washington, Publicación Científica 348; 1977. 


\section{Sobre as Autoras ${ }^{b}$}

15. Organización Panamericana de la Salud, Organización Mundial de un Grupo de Estudio en Ciencias de la Salud; 1978.

16. Jiménez K. A política de saúde escolar. sistema integral de saúde seguro escolar gratuito na ótica das enfermeiras do hospital belén de trujillo região la libertad-perú. [tese doutorado] Rio de Janeiro (RJ): Escola de Enfermagem Anna Nery/ UFRJ; 2003.

17. Organización Panamericana de la Salud, Organización Mundial de la Salud. Enseñanza de enfermería en salud comunitaria. Washington: Publicación. Científica 332; 1976.

18. Organización Panamericana de la Salud, Organización Mundial de la Salud La epidemiología y la enfermería. Washington: Publicación Científica 333; 1976.

19. Carvalho V. A Enfermagem de Saúde Pública como prática social: um ponto de vista crítico sobre a formação da enfermeira em nível de graduação. Esc Anna Nery Rev Enferm 1997 julho; 1 ( $n^{0}$ especial).

20. KosikK. Dialética do concreto. 2a. ed. Rio de Janeiro(R)): Paze Terra; 1995.

21. Bachelard G. A formação do espírito cientifico. Contribuição para uma análise do conhecimento. Rio de Janeiro (RJ): Contraponto; 1999.

22. Tavares L. Ajuste neoliberal e desajuste social na América latina. [tese doutorado] Campinas(SP): Instituto de Economia/UNICAMP; 1995

23. Manfred MA. Desarrollo a escala humana. Conceptos, aplicaciones y algunas reflexiones. Barcelona: Editorial Içaria; 1994.

\section{Notas}

aPresidente del Perú, aun cuestionado moral y políticamente, de nacionalidad indefinida aun.

\section{${ }^{\mathrm{b}}$ Adscripción Institucional:}

Profesoras de Tiempo Completo, perfil PROMEP. Integrantes de las Diferentes Líneas de Generación y Aplicación al Conocimiento del Cuerpo Académico de Enfermería en Salud Comunitaria. Promep. Facultad de Enfermería Victoria - Universidad Autónoma de Tamaulipas. México.

\section{Kelly Myriam Jiménez de Aliaga}

Dra. en Enfermería. Maestra en Enfermería en Salud Familiar y Comunitaria. Investigadora FEV - UAT. Líder de este Cuerpo Académico, perfil PROMEP.

\section{Cinthya Patricia Ibarra González}

Enfermera. Maestra en Ciencias de la Salud. Doctoranda "Enfermería en la Cultura de los Cuidados, perfil PROMEP"

\section{Olga Lidia Banda González}

Enfermera. Maestra en Educación. Doctoranda "Enfermería en la Cultura de los Cuidados", perfil PROMEP

\section{María Guadalupe Vásquez Salazar}

Enfermera. Maestra en Ciencias de la Salud. Doctoranda "Enfermería en la Cultura de los Cuidados"

\section{María Guadalupe Vásquez Treviño}

Enfermera. Maestra en Ciencias de la Salud. Doctoranda "Enfermería en la Cultura de los Cuidados"

\section{Maria Ramona Medrano Martínez}

Enfermera. Maestra en Ciencias de la Salud. Doctoranda "Enfermería en la Cultura de los Cuidados", perfil PROMEP. 